\title{
Comparative genomics on HHIP family orthologs
}

\author{
YURIKO KATOH $^{1}$ and MASARU KATOH ${ }^{2}$ \\ ${ }^{1}$ M\&M Medical BioInformatics, Hongo 113-0033; ${ }^{2}$ Genetics and Cell Biology Section, \\ National Cancer Center Research Institute, Tokyo 104-0045, Japan
}

Received October 19, 2005; Accepted November 18, 2005

\begin{abstract}
Hedgehog, FGF, VEGF, and Notch signaling pathways network together for vascular remodeling during embryogenesis and carcinogenesis. HHIP1 (HHIP) is an endogenous antagonist for $\mathrm{SHH}, \mathrm{IHH}$, and DHH. Here, comparative integromics analyses on HHIP family members were performed by using bioinformatics and human intelligence. HHIP1, HHIP2 (HHIPL1 or KIAA1822) and HHIP3 (HHIPL2 or KIAA1822L) constitute human HHIP gene family. Rat Hhip1, Hhip2, and Hhip3 genes were identified within AC107504.4, AC094820.6, and AC134264.2 genome sequences, respectively. HHIP-homologous (HIPH) domain with conserved 18 Cys residues was identified as the novel domain conserved among mammalian HHIP1, HHIP2, and HHIP3 orthologs. HHIPI mRNA was expressed in coronary artery endothelial cells, prostate, and rhabdomyosarcoma. HHIP 2 mRNA was expressed in trabecular bone cells. HHIP3 mRNA was expressed in testis, thyroid gland, osteoarthritic cartilarge, pancreatic cancer, and lung cancer. Promoters of HHIP family genes were not well conserved between human and rodents. Although GLI-, CSL-, and HES/HEY-binding sites were not identified, eleven bHLH-binding sites were identified within human $H H I P 1$ promoter. Expression of HES/ $H E Y$ family members, including HES1, HES2, HES3, HES4, HES5, HES6, HES7, HEY1, HEY2 and HEYL, in coronary artery endothelial cells was not detected in silico. Up-regulation of HHIP1 due to down-regulation of Notch-CSL-HES/HEY signaling cascade repressing bHLH transcription factors results in down-regulation of the Hedgehog-VEGF-Notch signaling cascade. On the other hand, down-regulation of HHIP1 due to up-regulation of Notch signaling in vascular endothelial cells during angiogenesis results in up-regulation of the Hedgehog-VEGF-Notch signaling cascade. Because HHIP1 is the key molecule for vascular remodeling, HHIP1 is the
\end{abstract}

Correspondence to: Dr Masaru Katoh, Genetics and Cell Biology Section, National Cancer Center Research Institute, 5-1-1 Tsukiji, Chuo-ku, Tokyo 104-0045, Japan

E-mail: mkatoh@ncc.go.jp

Key words: bioinformatics, comparative genomics, comparative proteomics, Hedgehog, VEGF, Notch, integrome network pharmacogenomics target in the fields of oncology and vascular medicine.

\section{Introduction}

Hedgehog signaling pathway is implicated in a variety of processes during embryogenesis, chronic persistent inflammation, and carcinogenesis (1-3). Hedgehog family of secreted proteins consists of Sonic Hedgehog (SHH), Indian Hedgehog (IHH) and Desert Hedgehog (DHH) (4-6). PTCH1, PTCH2, DISP1, DISP2 and DISP3 are multitransmembrane proteins with two PTCH/DISP homologous domains $(7,8)$. PTCH1 and PTCH2 are Hedgehog receptors, regulating the Hedgehog signal transducer Smoothened (SMO) (9-13). KIF27, KIF7, STK36, SUFU and DZIP1 are Hedgehog signaling components (10-17). GLI family transcription factors are implicated in the transcriptional activation of Hedgehog target genes, such as PTCHI, CCND2, IGFBP6, and FOXM1 (1,18-20).

HHIP1 (HHIP) is secreted-type Hedgehog-interacting protein, functioning as an endogenous antagonist for $\mathrm{SHH}$, $\mathrm{IHH}$, and DHH (21). HHIPI expression is down-regulated in a variety of tumors, such as gastric, pancreatic, colorectal, esophageal and lung cancer $(22,23)$. HHIPI down-regulation in pancreatic cancer is due to epigenetic $\mathrm{CpG}$ hypermethylation of HHIP1 promoter.

In contrast to the down-regulation of $H H I P 1$ in various types of human tumors, HHIP1 is abundantly expressed in human aortic endothelial cells (22). However, mechanism of HHIP1 expression in human aortic endothelial cells remains unclear.

Recently, we identified two other members of human HHIP gene family. Here, comparative genomics analyses on HHIP family members as well as expression analyses of HHIP family members were performed. Because HHIPI was expressed in coronary artery endothelial cells, transcriptional mechanism of HHIPI in coronary artery endothelial cells was further investigated.

\section{Materials and methods}

Identification of novel genes. Mouse cDNAs, ESTs, and rat genome sequences homologous to human HHIP1, HHIP2, and HHIP3 were searched for with the BLAST program (http:// www.ncbi.nlm.nih.gov) as described previously $(24,25)$. Exonintron boundaries were determined by examining the consensus sequence of exon-intron junctions ('gt ..... ag' rule of intronic 


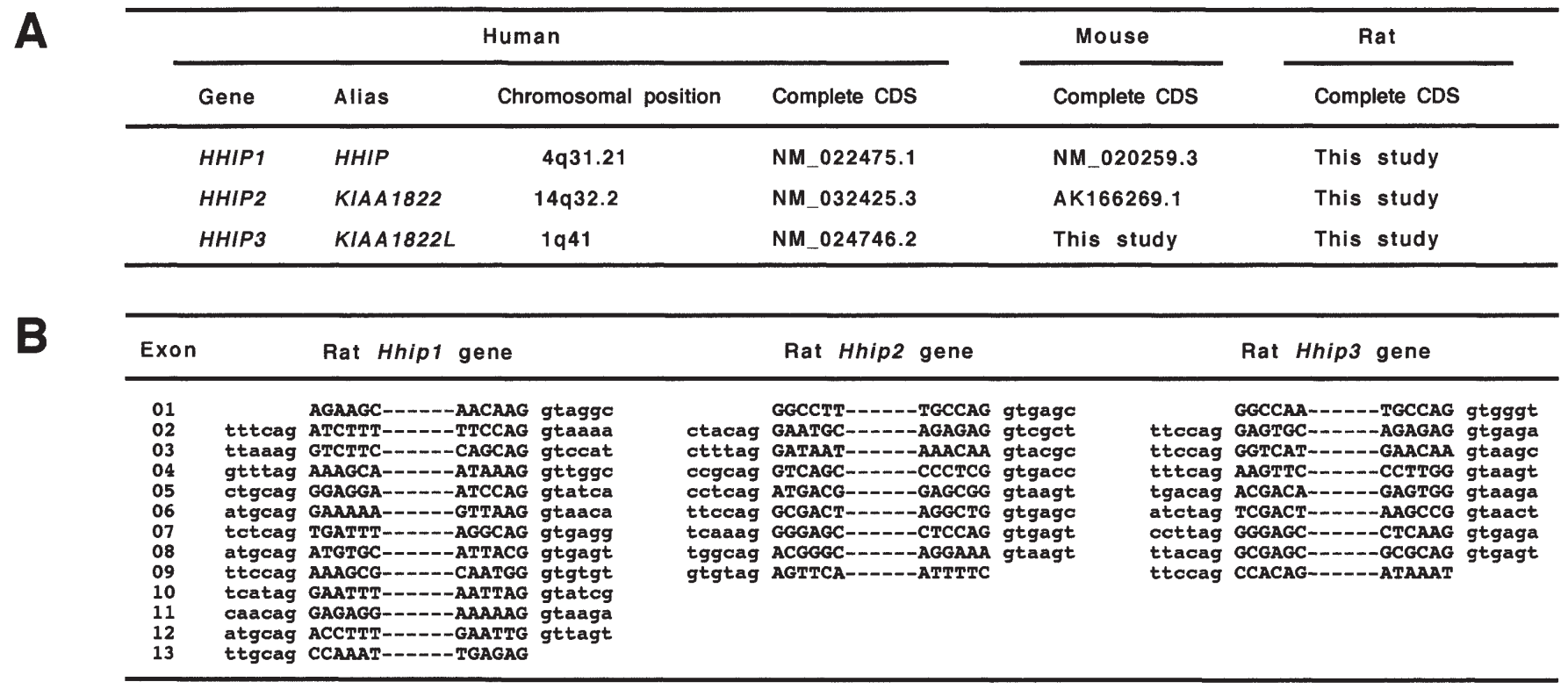

Figure 1. (A) Mammalian HHIP gene family consisting of HHIP1, HHIP2 and HHIP3 orthologs. (B) Exon-intron structure of rat Hhip1, Hhip2, and Hhip3 genes.

sequence) and the codon usage within the coding region as described previously $(26,27)$.

Comparative proteomics analyses. Translation into amino-acid sequence and amino-acid alignment were performed with the Genetyx program as described previously $(28,29)$. Signal peptide and transmembrane domain were searched for with the Kyte and Doolittle hydrophobicity analysis and PSORT II program. Domain architecture was at first searched for with the RPS-BLAST program (http://www.ncbi.nlm.nih.gov) as described previously $(30,31)$. Novel domains were then searched for based on the conservation among related proteins as described previously $(32,33)$.

Comparative genomics analyses. Genome sequences around human HHIP1, HHIP2 and HHIP3 genes were used as query sequences for the BLAST program to identify evolutionarily conserved regions. Transcription factor-binding sites within the promoter region were searched for with the Match program (http://www.gene-regulation.com) as well as by manual inspection.

\section{Results}

Human HHIP family genes. BLAST program using HHIP1 amino-acid sequence NP_071920.1 as a query sequence revealed that NM_032425.3 and NM_024746.2 RefSeqs were derived from human HHIP1-related genes. Human genes corresponding to NM_032425.3 and NM_024746.2 were designated HHIP2 (HHIPL1 or KIAA1822) and HHIP3 (HHIPL2 or KIAA1822L), respectively (Fig. 1A).

Preliminary alignment of HHIP family members revealed that 529-aa NP_079022.1 was N-terminally truncated HHIP3 protein. Although nucleotide position $644-2233$ of NM_ 024746.2 RefSeq was translated for NP_079022.1, we found that nucleotide position 59-2233 was the real coding region. Instead of 529-aa N-terminally truncated HHIP3 partial amino- acid sequence, 724-aa full-length HHIP3 amino-acid sequence translated from the real coding region of NM_024746.2 RefSeq was used for the following study.

Mouse Hhip family genes. Mouse cDNAs homologous to human HHIP1, HHIP2, and HHIP3 were searched for with BLAST programs. Mouse NM_020259.3 RefSeq, AK166269 cDNA, and NM_030175.1 RefSeq were derived from mouse Hhip1, Hhip2, and Hhip3 genes, respectively. NM_020259.3 RefSeq and AK166269 cDNA were representative full-length clones; however, NM_030175.1 RefSeq was a 5'-truncated partial clone (Fig. 1A).

BE305786 EST, corresponding to the 5'-UTR and Nterminal part of coding region of Hhip3, overlapped with NM_030175.1 5'-truncated partial RefSeq. Mouse Hhip3 complete CDS was determined by assembling BE305786 EST and 5'-truncated NM_030175.1 RefSeq (Fig. 1A).

Rat Hhip family genes. Rat Hhip1, Hhip2 and Hhip3 genes were identified within AC107504.4, AC094820.6 and AC134264.2 genome sequences, respectively. Exon-intron boundaries of rat Hhip1, Hhip2 and Hhip3 genes were determined by examining the consensus sequence of exon-intron junctions and the codon usage. Rat Hhip1, Hhip2 and Hhip3 genes were found to consist of 13, 9, and 9 exons, respectively (Fig. 1B). Complete CDSs of rat Hhip1, Hhip2 and Hhip3 were determined by assembling exonic regions. Rat Hhip1, Hhip2 and Hhip3 genes were found to encode 700-, 791- and 712-amino-acid proteins, respectively (Fig. 2).

Comparative proteomics on HHIP family members. Membrane topology analyses were performed at first. HHIP1 and HHIP2 orthologs were secreted proteins with $\mathrm{N}$-terminal signal peptide, while HHIP3 orthologs were type II transmembrane proteins with short N-terminal cytoplasmic region.

Human, rat, and mouse HHIP family members were then aligned for comparative proteomics analyses. Although $\mathrm{N}$ - and 


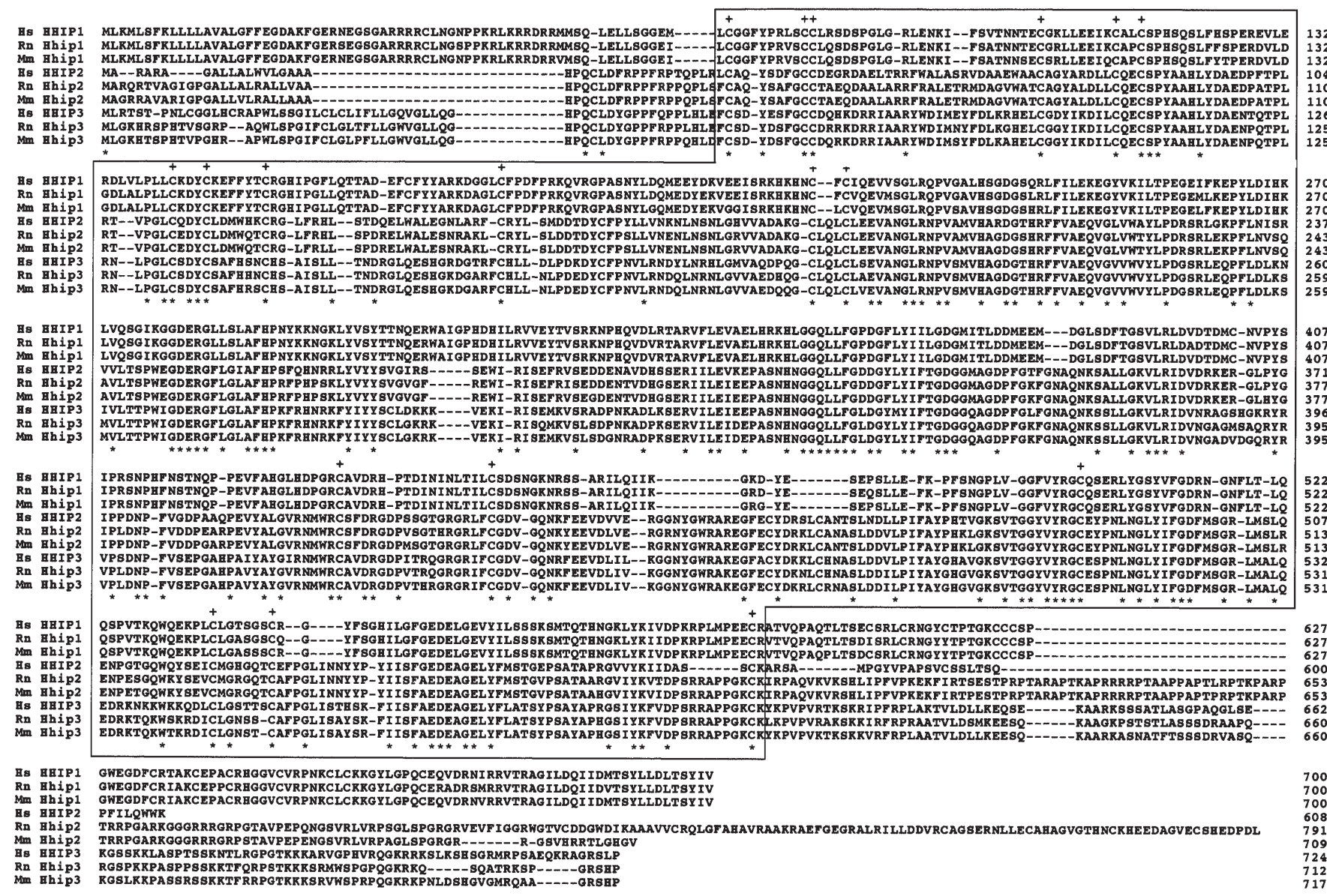

Figure 2. Alignment of vertebrate HHIP1, HHIP2 and HHIP3 orthologs. Hs, human. Rn, rat. Mm, mouse. HHIP homologous (HIPH) domain is boxed. Conserved Cys residues within the HIPH domain are shown by a cross.

C-terminal region of HHIP family members were divergent, core region corresponding to codon 68-595 of human HHIP1 was well conserved among mammalian HHIP family members (Fig. 2). The novel conserved region with 18 conserved Cys residues was designated the HHIP-homologous (HIPH) domain. These facts indicate that HHIP family members should be characterized as HIPH domain proteins.

Differential expression of HHIP1, HHIP2 and HHIP 3 mRNAs. ESTs corresponding to HHIP1, HHIP2, and HHIP3 mRNAs were searched for by using the BLAST program. The sources of ESTs were then listed up for the in silico expression analyses. HHIP1 mRNA was expressed in coronary artery endothelial cells, prostate, and rhabdomyosarcoma. HHIP2 mRNA was expressed in trabecular bone cells. HHIP 3 mRNA was expressed in testis, thyroid gland, osteoarthritic cartilarge, pancreatic cancer, and lung cancer.

Comparative genomics on HHIP 1, HHIP 2 and HHIP 3 orthologs. BLAST program as well as in house alignment of 5'-flanking regions revealed that HHIP1, HHIP2 and HHIP3 promoters were not well conserved between human and rodents.

Transcriptional regulation of human HHIP1 in coronary artery endothelial cells. We next analyzed the human HHIPI promoter to elucidate the mechanism for HHIP1 expression in coronary artery endothelial cells. GLI family transcsription factors, TCF/LEF family transcription factors, and CSL transcription factor are implicated in the transcriptional regulation of Hedgehog, WNT, and Notch target genes, respectively $(1,34-38)$. Because GLI-, TCF/LEF-, and CSLbinding sites were not identified within the human HHIPl promoter (Fig. 3A), HHIPl was not the direct transcriptional target gene of Hedgehog, WNT, and Notch signaling pathways.

Among $H E S / H E Y$ family genes encoding transcriptional repressors with bHLH and orange domains, including HES1, HES2, HES3, HES4, HES5, HES6, HES7, HEY1, HEY2 and HEYL $(39,40)$, at least HES1, HES5, HES7, HEY1, HEY2 and $H E Y L$ are best characterized Notch target genes. Expression of $H E S / H E Y$ family members was not detected in coronary artery endothelial cells by using in silico expression analyses.

Eleven bHLH-binding E-boxes were identified within human HHIPl promoter, while HES/HEY-binding N-box was not identified within human HHIP1 promoter (Fig. 3A). Because HES/HEY transcription factors repress bHLH factors, down-regulation of HES/HEY expression leads to up-regulation of HHIP1 mRNA depending on bHLH transcription factors (Fig. 3B).

\section{Discussion}

Mammalian HHIP family members were comprehensively identified and characterized in this study (Fig. 1). Complete 


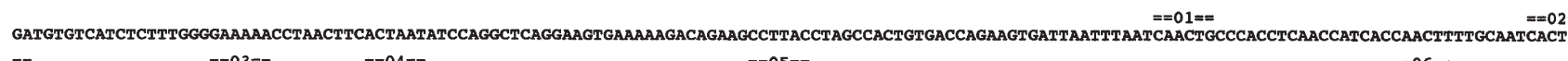

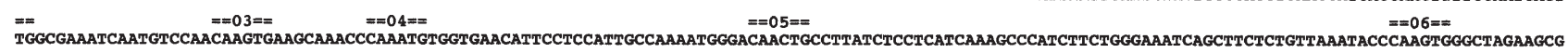
CATTTTGTGGGCAACCCTATAGAAGCTCGTCCTGATGCCAACTTGCAGGGATCCAGTAAACCTCAAGTCACCGCTCCTGATAACGTAGACATCTTTTTAGACACACTATTTMCACAGACTTTGTCTAGCTGGGGAAAAGGTTAATTTACT TGTATACACGGGGATAAGTAGAGCACAGCGCTTCTGGATGCCCTCAGACTTGCATCTAATTCTTTCTCCAGATCTAACGCCTCTGTTTCTACACCCAGGGAACCTCTCCCCAGGGACTGCGCGCGCGTGCGTGCAGGTGTGTGTGTGTG TGTGTGTGTGTGTGTGTTTGTGTGTGTTAAAACGAACACTAATTCCGCGCCCAGCTTCTCTCTCCGTCCACCTGCGGCCCAAGAACTTGTGTTCCCAGATGGTACCAGCGG $=08=09=10==$ Th $==11==$

CAAACCATCTCAGCCTACTCAACGGCATCTGGGATGTCCCCCTGCCTCTAAGTCAGACCCCAAGAAAGATCTTTTGAGGACTTGAGCTTCACGTTGAAAATCAGTGTGGAAAATGGGGGTGTCAGTGCGGCTGGGGGAGACAAAGGGGCCC AAAAGCTCCCTCCCGTCCGCTCCAGCCCACTTAGTTCACCCTCCCACGGGGCGAGGGGCGGGCCGGGGGGCTGAGGTGGGGATGGCCCTGGAAGGGGTCCCTCCCTTCCCACCTCCTACGGCCCACTCTTACTTACTTTCTTTCGGAGCC TCAGCTTGGCCGCCTTTAGCCCAGGCAGTGCGGAGGGGAGCGGGGAGGAGAGAGGAGCCTGCGGGCTGCAGCAGCCGGGCAGTCCCGGAATCTCCGGACTGCGTCCCTGCCCCAAGCCCGCCGGGAACCCGGGCCGTGGACGCCCCCAC CCGGGGGCGCGGCGAGGCGGGCGCGCGGGGTTCAGCACCCTCGAGGCTGGTTCCCGAAGCTCGCGGCCCTCCCCCTCCGTCCGCCGCCGTCGCGGCTAGCCGCCCCTCCCCCGCCGCAGCAGGGAGGCCGCCGGGGCCCGGGGGACGCGC TCGCGCGGGGCCGCCCCCTCCCCTTCCCTCCACCCTGGGCGGGGGCGGCGAGAAGCGTTGACGTCAAGGGGCGCGTTGTGGCAGCACCTCCCCGCGCGCTAGTTAAAAAGAAGAAGAAAAGAGGGAACGAAACATGAGAGGCTGTGTG

B

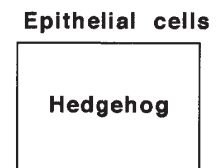

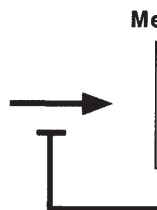

Mesenchymal cells

Endothelial cells

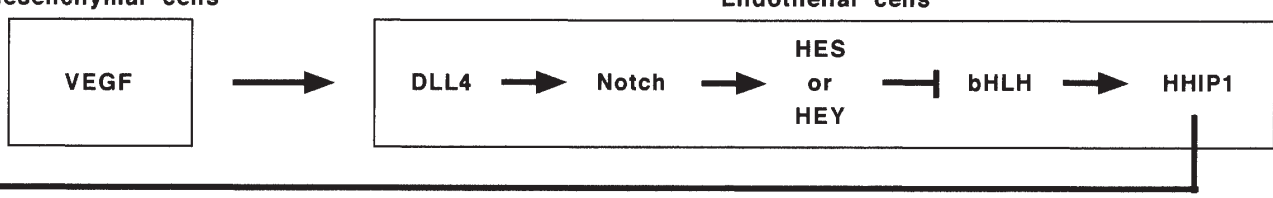

Figure 3. Regulation of HHIP1 transcription in vascular endothelial cells. (A) Human HHIP1 promoter. Exon 1 of human HHIP1 gene is boxed. Eleven bHLHbinding sites within human HHIP1 promoter are shown by double over-lines. (B) HHIP1 and Hedgehog-VEGF-Notch signaling cascade. Up-regulation of HHIP1 due to down-regulation of Notch-CSL-HES/HEY signaling cascade repressing bHLH transcription factors results in down-regulation of the Hedgehog-VEGFNotch signaling cascade. On the other hand, down-regulation of HHIP1 due to up-regulation of Notch signaling in vascular endothelial cells during angiogenesis results in up-regulation of the Hedgehog-VEGF-Notch signaling cascade.

CDS of mouse Hhip3 was determined by assembling BE305786 EST and 5'-truncated NM_030175.1 RefSeq. Complete CDS of rat Hhip1, Hhip2, and Hhip3 were determined by assembling exonic regions within AC107504.4, AC094820.6, and AC134264.2 rat genome sequences, respectively (Fig. 1B). Comparative proteomics analyses revealed that HIPH domain with 18 conserved Cys residues was conserved among mammalian HHIP1, HHIP2, and HHIP3 orthologs (Fig. 2).

HHIP1 mRNA was expressed in coronary artery endothelial cells, prostate, and rhabdomyosarcoma. HHIP2 mRNA was expressed in trabecular bone. HHIP3 mRNA was expressed in testis, thyroid gland, osteoarthritic cartilarge, pancreatic cancer, and lung cancer. Because preferential expression of HHIPl mRNA in coronary artery endothelial cells was the most interesting fact obtained by expression analyses, transcriptional mechanism of HHIP1 mRNA in coronary artery endothelial cells was further investigated.

Notch signaling pathway is implicated in artery morphogenesis during embryogenesis as well as angiogenesis during carcinogenesis $(34,35)$, and HES1, HES5, HES7, HEY1, HEY2, and $H E Y L$ are Notch target genes in vascular endothelial cells. Although we can not completely deny the false negativity based on in silico expression analyses, expression of HES/ $H E Y$ family members in coronary artery endothelial cells was not detected in this study. These facts indicate that the NotchCSL-HES/HEY signaling cascade was down-regulated in human coronary artery endothelial cells.

HES/HEY family members are repressors for bHLH transcription factors. Eleven bHLH-binding sites were identified within human HHIPI promoter (Fig. 3A), five bHLH-binding sites within rat Hhipl promoter, and two bHLH-binding sites within mouse Hhipl promoter. Down-regulation of the NotchCSL-HES/HEY signaling cascade in coronary artery endothelial cells leads to HHIPI up-regulation depending on bHLH transcription factors (Fig. 3B).
VEGF-induced expression of DLL4 in vascular endothelial cells leads to the activation of Notch signaling (41). Notch signaling activation leads to up-regulation of HES/ HEY family members, and the following down-regulation of HHIP1 (Fig. 3B). HHIP1 down-regulation then leads to SHH activation (21), which results in the activation of VEGF signaling (42). Therefore, VEGF-induced downregulation of HHIP1 during angiogenesis leads to the positive feedback to the Hedgehog-VEGF-Notch signaling cascade (Fig. 3B).

Expression level of HHIP1 affects vascular remodeling through the regulation of Hedgehog-VEGF-Notch signaling cascade. HHIP1 itself could be utilized for anticancer agent as the Hedgehog inhibitor. On the other hand, monoclonal antibody, RNAi compound, and small-molecule compound down-regulating HHIP1 function could enhance the angiogenic effects of VEGF and FGFs for coronary artery disease. HHIP1 is the pharmacogenomics target in the fields of oncology and vascular medicine.

\section{References}

1. Katoh Y and Katoh M: Hedgehog signaling in gastric cancer. Cancer Biol Ther 4: 1050-1054, 2005.

2. Van den Brink GR, Bleuming SA, Hardwick JC, et al: Indian Hedgehog is an antagonist of Wnt signaling in colonic epithelial cell differentiation. Nat Genet 36: 277-282, 2004.

3. Garciadiego-Cazares D, Rosales C, Katoh M and ChimalMonroy J: Coordination of chondrocyte differentiation and joint formation by $\alpha 5 \beta 1$ integrin in the developing appendicular skeleton. Development 131: 4735-4742, 2004.

4. Marigo V, Roberts DJ, Lee SM, et al: Cloning, expression, and chromosomal location of $\mathrm{SHH}$ and $\mathrm{IHH}$ : two human homologues of the Drosophila segment polarity gene hedgehog. Genomics 28: 44-51, 1995 .

5. Katoh Y and Katoh M: Identification and characterization of rat Desert hedgehog and Indian hedgehog genes in silico. Int J Oncol 26: 545-549, 2005.

6. Katoh Y and Katoh M: Comparative genomics on Sonic hedgehog orthologs. Oncol Rep 14: 1087-1090, 2005. 
7. Johnson RL, Rothman AL, Xie J, et al: Human homolog of patched, a candidate gene for the basal cell nevus syndrome. Science 272: 1668-1671, 1996.

8. Katoh Y and Katoh M: Identification and characterization of DISP3 gene in silico. Int J Oncol 26: 551-556, 2005.

9. Xie J, Murone M, Luoh SM, et al: Activating Smoothened mutations in sporadic basal-cell carcinoma. Nature 391: 90-92, 1998.

10. Hooper JF and Scott MP: Communicating with Hedgehogs. Nat Rev Mol Cell Biol 6: 306-317, 2005.

11. Briscoe $\mathrm{J}$ and Therond $\mathrm{P}$ : Hedgehog signaling: from the Drosophila cuticle to anti-cancer drugs. Dev Cell 8: 143-151, 2005.

12. Lum L and Beachy PA: The Hedgehog response network: sensors, switches, and routers. Science 304: 1755-1759, 2004.

13. Pasca di Magliano M and Hebrok M: Hedgehog signalling in cancer formation and maintenance. Nat Rev Cancer 3: 903-911, 2003.

14. Katoh Y and Katoh M: KIF27 is one of orthologs for Drosophila Costal-2. Int J Oncol 25: 1875-1880, 2004.

15. Katoh Y and Katoh M: Characterization of KIF7 gene in silico. Int J Oncol 25: 1881-1886, 2004.

16. Taylor MD, Liu L, Raffel C, et al: Mutations in $S U F U$ predispose to medulloblastoma. Nat Genet 31: 306-310, 2002.

17. Moore FL, Jaruzelska J, Dorfman DM and Reijo-Pera RA: Identification of a novel gene, DZIP (DAZ-interacting protein), that encodes a protein that interacts with DAZ (deleted in azoospermia) and is expressed in embryonic stem cells and germ cells. Genomics 83: 834-843, 2004.

18. Kinzler KW, Bigner SH, Bigner DD, et al: Identification of an amplified, highly expressed gene in a human glioma. Science 236: 70-73, 1987.

19. Teh MT, Wong ST, Neill GW, et al: FOXM1 is a downstream target of Gli1 in basal cell carcinomas. Cancer Res 62: 4773-4780, 2002.

20. Yoon JW, Kita Y, Frank DJ, et al: Gene expression profiling leads to identification of GLI1-binding elements in target genes and a role for multiple downstream pathways in GLI1-induced cell transformation. J Biol Chem 277: 5548-5555, 2002.

21. Chuang PT and McMahon AP: Vertebrate Hedgehog signalling modulated by induction of a Hedgehog-binding protein. Nature 397: 617-621, 1999.

22. Olsen CL, Hsu PP, Glienke J, Rubanyi GM and Brooks AR: Hedgehog-interacting protein is highly expressed in endothelial cells but down-regulated during angiogenesis and in several human tumors. BMC Cancer 4: 43, 2004.

23. Martin ST, Sato N, Dhara S, et al: Aberrant methylation of the human Hedgehog interacting protein (HHIP) gene in pancreatic neoplasms. Cancer Biol Ther 4: 728-733, 2005.
24. Katoh M: Paradigm shift in gene-finding method: from benchtop approach to desk-top approach. Int J Mol Med 10: 677-682, 2002.

25. Katoh M and Katoh M: $C L D N 23$ gene, frequently down-regulated in intestinal-type gastric cancer, is a novel member of CLAUDIN gene family. Int J Mol Med 11: 683-689, 2003.

26. Katoh $M$ and Katoh M: Identification and characterization of human MPP7 gene and mouse Mpp7 gene in silico. Int J Mol Med 13: 333-338, 2004

27. Katoh $M$ and Katoh M: Identification and characterization of Crumbs homolog 2 gene at human chromosome 9q33.3. Int J Oncol 24: 743-749, 2004

28. Katoh $\mathrm{M}$ and Katoh $\mathrm{M}$ : Identification and characterization of human CKTSF1B2 and CKTSF1B3 genes in silico. Oncol Rep 12: 423-427, 2004.

29. Katoh $Y$ and Katoh M: Identification and characterization of rat Wnt6 and Wnt10a genes in silico. Int J Mol Med 15: 527-531, 2005.

30. Katoh Y and Katoh M: Comparative genomics on SFRP1 orthologs. Int J Oncol 27: 861-865, 2005.

31. Katoh M and Katoh M: Comparative genomics on SFRP2 orthologs. Oncol Rep 14: 783-787, 2005.

32. Katoh Y and Katoh M: Comparative genomics on DKK1 orthologs. Int J Oncol 27: 275-279, 2005.

33. Katoh Y and Katoh M: Comparative genomics on DKK2 and DKK4 orthologs. Int J Mol Med 16: 477-481, 2005.

34. Radtke F and Raj K: The role of Notch in tumorigenesis: oncogene or tumour suppressor? Nat Rev Cancer 3: 756-767, 2003.

35. Li JL and Harris AL: Notch signaling from tumor cells: a new mechanism of angiogenesis. Cancer Cell 8: 1-3, 2005.

36. Katoh M: Regulation of WNT signaling molecules by retinoic acid during neuronal differentiation in NT2 cells: threshold model of WNT action (Review). Int J Mol Med 10: 683-687, 2002.

37. Katoh M: $W N T$ and $F G F$ gene clusters (Review). Int J Oncol 21: $1269-1273,2002$.

38. Katoh M: WNT2B: comparative integromics and clinical applications (Review). Int J Mol Med 16: 1103-1108, 2005.

39. Katoh $M$ and Katoh M: Identification and characterization of human HES2, HES3, and HES5 genes in silico. Int J Oncol 25: 529-534, 2004

40. Katoh $\mathrm{M}$ and Katoh $\mathrm{M}$ : Identification and characterization of human HESL, rat Hesl and rainbow trout hesl genes in silico. Int J Mol Med 14: 747-751, 2004.

41. Patel NS, Li JL, Generali D, et al: Up-regulation of Delta-like 4 ligand in human tumor vasculature and the role of basal expression in endothelial cell function. Cancer Res 65: 8690-8697, 2005.

42. Lawson ND, Vogel AM and Weinstein BM: Sonic hedgehog and vascular endothelium growth factor act upstream of Notch pathway during arterial endothelial differentiation. Dev Cell 3: 127-136, 2002. 